(24 St. Fasten). 570 mal vergrössert. Randzellencomplex. Text pag. 451, Anmerk. 3.

Fig. 4. Tubulus-Durchschnitt aus der durch Pilocarpininjection gereizten Submaxillardrüse einer Katze. 420 mal vergrössert. Text pag. 453.

Fig. 5. Ebendaher, $420 \mathrm{mal}$ vergrössert.

Fig. 6. Durchschnitt durch einen Tubulus einer stark gereizten Unterkieferdrüse eines Hundes. 560 mal vergrössert. Copie aus Stöhr (21). Tafel XVII, Fig. 4.

Fig. 7. Tubulus-Durchschnitt von dem gleichen Objekt wie Fig. 4. $420 \mathrm{mal}$ vergrössert. Text pag. 454, Anmerk.

Fig. 8. Durchschnitt durch eine Speichelröhre, ebendaher. $1100 \mathrm{mal}$ vergrössert. Zeiss' homog. Immersion. Text pag. 455.

Fig 9. Tubulus-Durchschnitt ebendaher. 1100 mal vergrössert. Der Sehnitt ist nur links ein senkrechter, unten und rechts ist er schräg durch die Schleimzellen gegangen und täuscht Secretcapillaren an der Basis der Schleimzellen vor. Text pag. 455.

(Aus dem ersten anatomischen Institut in Berlin.)

\title{
Ueber die sympathischen Ganglienzellen der Nager.
}

$$
\text { Von }
$$

\section{Dr. Hugo Apolant.}

Hierzu Tafel XXIII.

Die auffallende Thatsache, dass die Zellen der Grenzstrangganglien ausgewachsener Kaninchen mit so grosser Constanz zwei Kerne zeigen, hat in einigen Fällen Veranlassung gegeben, die Stellung von Zellen zweifelhafter Natur nach diesem Kriterium zu präcisiren. So mterscheidet $\mathrm{Vigna} \mathbf{1}^{1}$ ) in den Herzganglien des Kaninchens anipolare Zellen mit nur einem Kern und multipolare mit stets zwei Kernen. Die ersteren hält er für cerebro-

1) Vignal, Gazette médic. de Paris. 1880. Nr. 49. 
spinal, die letzteren für sympathisch. Leider fehlen Angaben über die Untersuchungsmethode und das Alter der untersuchten Thiere. In noch bestimmterer Weise, ohne die Polarität der Zellen zu berücksichtigen, glaubt $L$ angendo $\operatorname{rf}^{1}$ ) als unterstützenden Beweis für die spinale Natur des Gangl. ciliare die Thatsache anführen zu dürfen, dass die Zellen des Ciliarknotens beim Kaninchen stets nur einen Kern enthalten. Ich will mich an dieser Stelle nicht weiter tiber die Bedeutung und Stellung des Gangl. ciliare verbreiten, weil ich diese viel umstrittene Frage demnächst in einer speciellen Arbeit auf Grund besonderer Methoden zu erörtern beabsichtigte. Nur darauf möchte ich hinweisen, dass die Beweisfthlung Langend orff's im Widerspruch steht mit den Ergebuissen der Golgi'schen Methode, nach denen, wie die Untersuchungen von Retziusº, d'Er-

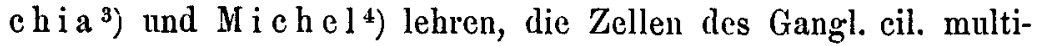
polar sind und vollkommen den Charakter sympathischer Zellen haben.

Es war daher \%u untersuchen, ob bein Kaninchen dem Vorbandensein zweier Kerne eine höhere Beweiskraft ftir die Bestimmung der sympathischen Natur der Ganglienzellen innewohnt als der Multipolarität.

Auf Anregung von Herrn Geheimrath Waldeyer habe ich daher an zahlreichen Kaninchen verschiedenen Alters die Ganglien des Grenzstranges vom Gangl. cervic. suprem. bis $\% u$ den Sacralganglien mit Einschluss der grossen Luterleibsganglien (Gangl. coeliac., mesent. inf. u. lypogastr.) sowie des kleinen oberhalb des Arcus Aortae gelegenen Gangl. cardiac., ferner von den Kopfganglien das Gangl. ciliare, sphenopalat., submaxill. und die beiderseits doppelt vorhandenen Ganglia otica untersucht.

Trotzdem die Untersuchungsreihen ziemlich ausgedehnt waren, kann ich mich bei der Mittheilung der Resultate relativ kurz fassen, da es sich weniger um histologische Feinheiten als um die Feststellung leicht eruirbarer thatsächlicher Verhältnisse handelt.

1) Langend orff, Ciliarganglion und oculomotorius. P fl ü ger's Arch. Bd. 56.

2) Retzius, Gangl. cil. Biolog. Untersuch. N. F. VI.

3) d'Erchia, Contrib. dello studio ete. Monit. zool. ital. 31. 8. 1894.

4) Michel, Ueber die feinere Anat. d. Gangl. cil. Verhandl. d. achten internat. ophthalmol. Congr. in Edinburgh. 1894. 
Hierzu kommt, dass ein wesentlicher Theil meiner Untersuchungen sich lediglich als Nachprüfung, bezugsweise Bestätigung und Ergänzung der Schwalbe'schen Angaben herausgestellt hat.

Was die Methode betrifft, so halte ich noch immer frisches Zerzupfen in Jodserum für die beste. Weitans die grösste An. zahl meiner Präparate ist so hergestellt. Bei sebr alten Thieren ist allerdings eine längere Maceration wünschenswerth. Auch hierfür leistet nach meinen Erfahrungen schwaches, natürliches Jodserum, das täglich mit stärkerem nach $R$ anvier's ${ }^{1}$ ) Vorschrift jodirt wird, am meisten. Die Präparate leiden auch bei wochenlanger Maceration nicht und könuen stets bequem mit Carmin gefärbt werden. Salzsäureglycerin nach vorheriger Osmiumfixation ( $\mathrm{Schwalbe}{ }^{2}$ ) ist zwar für die Zellenisolation vorzuziehen, lat indessen den Nachtheil, die Kerne häufig bis zur Unkenntlichkeit zn verwischen.

Wie Schwalbe ${ }^{3}$ ) gezeigt hat, findet man in den Grenzstrangganglien und im Sympathicusstamm junger Kaninchen sowohl einkernige wie zweikernige Zellen. Mit vorrüekendem Alter vermindert sich die Zahl der einkernigen in demselben Maasse wie die der zweikernigen zunimint, so dass die ersteren als Vorstufe der letzteren anzusehen sind. Ich fugge hier ergänzend hinzu, dass sich zweikernige Zellen weit in das embryonale Leben hinein verfolgen lassen. Schon an Embryonen. in der dritten Woche sind sie an Schnittserien nachzuweisen. Ferner ist es mir mehrfach geglückt, bei drei Wochen alten Kaninchenembryonen das Gangl. cervic. supr. unter der Lupe zu präpariren und vorsichtig mit feinen Nadeh in Jodserum frisch 7.11 verzupfen. Wenn auch bei dieser Präparation wegen der Zartheit des Objectes die Zellform als solche oft verloren geht, so lassen sich doch schon an zahlreichen Zellen die beiden meist dicht an einanderliegenden Kerne erkennen (Fig. 5a).

Treten so einerseits die zweikernigen Zellen schon in der Mitte des embryonalen Lebens auf, so lassen sich andrerseits die einkernigen bis in das späte Alter der Thiere verfolgen. Wenn

1) Ranvier, Technisches Lehrbuch der Histol. S. 72.

2) Schwalbe, Ueber die Caliberverhältnisse der Nervenfasern. Leipzig 1882. S. 12.

3) Schwalbe, Ueber den Bau der Spiualganglien etc. Arch f. mikrosk. Anat. Bd. IV. 
ich Schwalbe recht verstehe, zühlt er alle einkernigen Zellen, die sich bei alten, ausgewachsenen Thieren finden, zu den von ihm zuerst beschriebenen bipolaren Zellen, die sich auch durch eine grössere Zartheit des Zellleibes vor den übrigen auszeichnen, während alle multipolaren Zellen in diesem Stadium zwei Kerne enthalten. In dieser Allgemeinheit kann ich den Schwal be'schen Angaben nicht zustimmen. Unter der grossen Anzahl von Ganglien, die ich in mehrmonatlichem Studium untersuchte, fand ich auch bei ganz alten Thieren, obschon selten, einkemige, evident multipolare Zellen, die sich in nichts von den analogen Bildungen junger Thiere unterscheiden. Fig. 3 zeigt cine solclie Zelle aus einem Gangl. lumbale eines zweijührigen, auffallend grossen Kaninchenbockes. Für das Gangl. cocliac. hat schon B id $\left(\mathrm{e} \mathrm{r}^{1}\right.$ ) das Vorkommen einkerniger Zellen auch bei ganz alten Thieren constatirt. Seiner Schlussfolgerung indessen, lass aus dem relativ häufigen Vorkommen einkerniger Zcllen \%wischen diesen und den zweikernigen nicht sowohl Alters- als functionelle Unterschiede sich ergeben, kann ich nicht zustimmen, da nach meiner Erfahrung einkernige Zellen bei alten Thieren selten sind und häufig durch lädirte zweikernige Zellen vorgetäuscht werden. Es sei hier übrigens auch erwähnt, dass ich dic Multipolarität dieser einkernigen Zellen, die von $\mathrm{Sch}$ wa l be als wahrscheinlich hingestellt wurde, auch bei jungen Thieren mehrfach nachweisen konnte, (s. Fig. 2).

Eine zweite Frage, ther die ich mich allerdings nicht in gleicher Weise positiv aussprechen kann, ist die, ob alle cinkernigen bipolaren Zellen auch bei alten Thieren einkernig bleiben, wie es Schwalbe anzunehmen scheint, oder ob derselbe Process, wie bei den multipolaren Zellen, auch hier stattfinden kann. Was mich veranlasst, diese Frage vorläufig noch für unentschieden zu halten, ist folgendes. Ich labe diese bipolaren Zellen im Sympathicusstamm junger Thiere nicht gar so selten gefunden, häufiger jedenfalls, als ich nach den Angaben Schwalbe's erwartet hatte. Ausdrücklich betone ich, dass ich diese Zellen nur dann als solche diagnosticirte, wenn die Bipolaritït so deutlich wie in der Abbildung Fig. 1 ausgesprochen war. Bei alten Thieren trifft man nun nach meinen Erfahrungen diese Zellen

1) Bidder, Die Nervi splanchnic. Arch. f. Anat. u. Phys. 1869. 
entschieden seltener. Es bleibt allerdings die Möglichkeit bestehen, dass dieser seltenere Fund bei alten Thieren anf einer durch die grössere Zähigkeit des Bindegewebes bedingten schwierigeren Isolirbarkeit der Zellen beruht. Andrerseits gelingt es hier öfter als bei jungen Thieren zweikernige Zellen $\% u$ isoliren, deren Bipolorität zuweilen höchst frappant ist. Freilich ist zu berücksichtigen, dass man in dieser Be\%iehung ausserordentlich leicht Täuschungen ausgesetzt sein kann, zumal die Zellen des Sympathicusstammes in sitı schon häufig eine langgestreckte bipolare Form haben. Ist doch $\mathrm{G}$ uye ${ }^{1}$ ) offenbar durch derartige Formen zu dem weitgehenden Schluss verleitet worden, alle sympathischen Zellen des Kaninchens für bipolar zu halten und die Bedeutung der beiden Kerne im Sinne Arnolds und Beales darin zu sehen, dass sie die Endigungen der Nervenfortsätze darstellen. Bei dem hentigen Stande der Technik düfte die obige Frage nur mit einer Methode zu lösen sein, die Kerne und Fortsätze in gleich guter Weise zur Anschauung bringt. Das leistet bis jetzt allein die vitale Methylenblaufärbung. Leider sind meine zahlreichen dicsbezüglichen Versuche nur in so unvollkommener Weise geglückt, dass ich sie zur Entscheidung der Frage nicht heranzieben kann.

Dieselben Verhältnisse wie in den Grenzstrangganglien walten in den oben erwähnten grossen Unterleibsganglien und in dem kleinen Gangl. cardiac., so dass ich auf diese nicht genauer einzugehen brauche. Erwähnen möchte ich hier nur eines gelegentlichen, äusserst seltenen Befundes von 3 Kernen, wie ihn Fig. 4 aus dem Gangl. coeliacum des Kaninchens darstellt.

Aus den bisher erwähnten Thatsachen geht hervor, dass das Gesetz der Zweikernigkeit der sympathischen Zellen auch bei alten Kaninchen nicht ausnahmslos besteht, und dass wir daher eine isolirte Zelle aus der Anzahl der Kerne allein nicht mit absoluter Sicherheit zu diagnosticiren vermögen. Hierzu kommt,

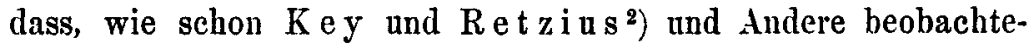
ten, sich gelegentlich auch in den Spinalganglien der Kaninchen Zellen mit zwei Kernen finden. Immerhin könnte man versucht

1) Guye, Centralbl. f. d. medic. Wissensch. 1866. Nr. 56.

2) Key und Retzius, Studien in der Anat. d. Nervensyst. der zweiten Hälfte. Stockholm 1876. 
sein, aus den bisherigen Daten den Schluss zu ziehen, dass bei alten Kaninchen in den sympathischen Ganglien die zweikernigen, in den spinalen dic einkernigen Zellen so überwiegen, dass hieraus die Natur des Ganglion eruirbar ist. Dieser Schluss ist indessen falsch, wie die Untersuchung der Kopfganglien lehrt. Ueber die Natur des Gangl. ciliar. herrscht allerdings noch keine völlige Einigkeit, dagegen wird, so weit mir bekannt, die sympathische Natur des Gangl. sphenopalat., otic. und submaxillare von keiner Seite angezweifelt. Alle genannten Ganglien zeigen nun aber stets, a u ch bei ganzalten Thiele $n$, $n$ ur einkernige $Z$ elle $n$. $Z$ ellen m it $2 \mathrm{~K}$ ernen sind hier ebensoselten wie in den Spinalganglien. Nur ein einziges Mal sah ich im Gangl. oticum eine zweikernige Zelle ${ }^{1}$ ). Damit fällt jede Berechtigung, die Natur des Ciliargangrlions aus der Anzahl der Zellkerne zu bestimmen.

Die diagnostísche Bedeutung der beiden Kerne verliert aber noch mehr an Boden damit, dass sich. unter den untersuchten Nagern nur noch beim $M$ eers $\mathrm{ch}$ weinclien, wo es schon $\mathrm{Schwalbe}$ constatirt hat, und beim $\mathrm{H}$ as en die gleichen Verhältnisse wie beim $K$ an in $\mathrm{ch}$ c n finden, während $R$ at $t \mathrm{e}, \mathrm{M}$ a us und Eich hörnchen fast ausschliesslich im ganzen sympathiscben System einkernige Zellen aufweisen. Ich bemerke ausdrücklich, dass von den zuletzt genannten überwiegend alte Thiere untersucht wurden, beim Eichliörnchen sogar so alte Exemplare, dass die Isolirung der Zellen erhebliche Schwierigkeiten bot. Es ist geradezu eine Ausnalıme, wenn man, was allerdings vorkommt, hier zweikernige Zellen findet. Ein derartiges gelegentliches Vorkommen zweikerniger Zellen ist aber auch bei andern Thieren, wie bei der Katze (B i d der ${ }^{2}$ ), beim Frosch (Cour v o i s i e r) ${ }^{3}$ ), beim Hund, Menschen etc. (M a y e r) ${ }^{4}$ ) mehrfach constatirt worden.

Ist somit die Zweikernigkeit sympathischer Zellen weder für die Nagethiere im Allgeneinen noch für das ausgewachsene

1) Uebrigens finde ich schon bei Retzius, Arch. f. Anat. $u$. Phys. 1880 eine Abbildung zweier Zellen aus dem Gangl, otic. des Kaninchens mit nur je einem Kern.

2) 1 . c.

3) Courroisier, Schultze's Arch. Bd. 2.

4) Mayer in Stricker's Handb. der Gewebelehre. 
Kaninchen im Besonderen ein absolut giltiges Gesetz und daher für die Diagnose von Ganglien zweifelhafter Natur nicht zu verwerthen, so bleibt doch das innerhalb der oberı gezeichneten Grenzen constante Vorkommen zweikerniger Zellen eine so auffallende Erscheinung, dass sich die Frage nach dem Zustandekommen beziehungsweise der Bedeutung der beiden Kerne aufdrängt.

Diese Frage ist bisher nur selten aufgeworfen und unvollkommen beantwortet worden. Gu y e's oben erwähnte Ansicht, dass die Kerne die Endigungen zweier Nervenfortsätze darstellen, ist bereits von $\mathrm{Schwalbe}$ als mit den Thatsachen im Widerspruch stehend zurückgewiesen worden. $\mathrm{B}$ i d d e ${ }^{1}$ ) hat vergeblich nach Momenten gesucht, die die sonderbare Erscheinung erklären könnten. Nur S $\mathrm{chwalbe}$ kommt der Lösung näher, indem el die Unmöglichkeit nachweist, dass es sich bei dem Zustandekommen der zwei Kerpe um eine unvollkommene Zelltheilung handelt; vielmehr entstehen die beiden Kerne nàch diesem Autor entweder durch Theilung des ursprïnglich einfachen Kerns oder clurch Verschmelzen 'wweier Zellen mit Beibehaltung der Kerne. Welcher dieser Vorgänge zul Recht besteht, lässt er indessen offen. Fassen wir zunächst die letztere Möglichkeit ins Auge, so müssen wir bekennen, dass eine Verschmelzung zweier Ganglienzellen im ausgebildeten Zustand unseren modernen Anschaungen erhebliche Schwierigkeiten bietet. Die Melrzahl der Autoren steht heute auf dem Standpunkt der Contactwirkung unter Leugnung der Ganglienzellanastomosen. Da nun die Bildung zweikerniger Zellen keineswegs nur an das embryonale Leben gebunden ist, sondern sicher auch zu einer Zeit stattfindet, in der die Zelle und das Neuron anatomisch und physiologisch als fertig anzusehen ist, ja vermuthlich bis in das höchste Alter der Thiere fortdauert, so milssten wir uns vorstellen, dass der ursprüngliche Contact in eine solide Anastomose mit folgender Verschmelzung der Zellkörper überginge. Diese Vorstellung hat aber sowohl anatomisch wie physiologisch ihre grossen Bedenken. Ich will allerdings nicht verschweigen, dass ich einmal, aber auch nur in einem einzigen Präparate vom Gangl. cervic. suprem. eines viermonatlichen Hasen anf zwei Zellen gestossen bin (siehe

1) l. c. 
Fig. 7 a und 8, die in der Mitte eine so eigenthümliche Einschnürung zeigten, dass man sie entweder als in Theilung oder als in einem Stadium der Verschmelzung begriffen ansehen möchte. Ich kann indessen auf diesen Befund, der ein gänzlich vereinzelter geblieben ist, kein Gewicht legren. Ebenso wenig beweisend sind die sogenannten Zellbrïckell, voll denen $\mathrm{Ma} \mathrm{yer}^{\circ}$ ) eine Abbildung giebt. Mir ist es niemals geglickt, etwas derartiges zu sehen, so dass ich kein eignes Urtheil über die Häufigkeit dieser Bildungen habe. Würden sie aber thatsächlich den Beginn einer Verschmelzung zweier Zellen darstellen, so müsste man bei der Bearbeitung eines so umfangreichen Materials, wie des zu dieser Arbeit verwandten, doch viel häufiger, als es der Fall war, auf vorgeschrittene Stadien der Verschmelzung stossen, die als solche, wenn auch nur mit einer gewissen Wahrscheinlichkeit zu deuten wären.

Einen anderen, sehr viel bedeutungsvolleren Befund, der weit eher zu Gunsten einer Zellenverschmelzung gedeutet werden könnte, erwähnt Ma yer, nämlich eine Zelle aus dem Gangl. coeliacum des Kaninchens, die ausser mehreren verästigrten Protoplasmafortsätzen zwei Axencylinderfortsätze zeigte, die sich unwcit von der Zelle mit Mark belegten. Es ist sehr bedauerlich, dass Mayer keine Abbildung dieses gewiss seltenen Befundes gegeben hat und ferner nicht erwähnt, ob die betreffende Zelle zwci Kerne gehabt hat. Im Gegensatz zu diesen ganz vereinzelt dastehenden Beobachtungen bin ich in der Lage, Thatsachen anzuführen, die ein allgemeinere Giltigkeit haben und uns zu einer anderen Auffassung der zweikernigen Zelle zwingen. Es zeigt sich nämlich, dass bei Kaninchen, Meerschweinchen und Hasen, deren Grenzstrangganglienzellen eine ausgesprochene Tendenz zur Bildung zweier Kerne zeigen, in der Grösse dieser zweikernigen Zellen ausserordentliche Verschiedenheiten bestehen, die mir bei jungen Thieren stärker ausgeprägt erscheinen als bei alten. Einen in dieser Beziehung extremen, obwohl durchaus nicht vereinzelten Fall illustrirt Fig. 7. Die beiden Zellen lagen im Präparat genau so nahe an einander wie es die Figur wiedergibt. Die grössere Zelle hat den stattlichen Längsdurchmesser von $126 \mu$, während der entsprechende Durchmesser der kleinen Zelle nur $25 \mu$ bè-

1) 1. c. 
trägt. Die Durclımesser der Zellkerne betragen 16 bezw. $6 \mu$. Würden die zweikernigen Zellen aus der Verschmelzung zweier einkerniger entstehen, so wäre es eine nothwendige Forderung, dass man in früheren Entwicklungsstadien einkernige Zellen fände, die böchstens halb so gross sind als die kleinsten zweikernigen. Zellen von so kleinen Dimensionen habe ich aber bei intaktem Zellleib nie gesehen. Ferner möchte ich auf eine Thatsache hinweisen, die ich öfter beobachtete, ohne indessen hieraus den Schluss auf ein allgemein giltiges Gesetz ziehen zu können. In jugendlichen einkernigen Zellen ist nämlich der Kern häufig so gross, dass er nur von einer relativ schmalen Protoplasmazone umgeben ist. Dies Missverhältniss giebt nicht selten, besonders beim Zerzupfen embryonaler Ganglien, zur Isolirung von Kernen Veranlassung, denen nur Spuren von Protoplasma anhaften. Bei selr kleinen zweikernigen Zellen ist die Gesammtmasse der Kerne allerdings auch sehr gross imVerhältniss zum Zelleib, mit demWachsthum des letzeren scheint aber der Kern nicht gleichen Schritt zu halten, so dass man zuweilen zwcikernige Zellen ron den doppelten Dimensionen einkerniger findet, während dieser eine Kern selbst grösser ist als jeder der beiden Kerne ersterer Zellen.

Diese Thatsachen machen es wahrscheinlich, dass die beiden Kerne durch Theilung des ursprünglich einfachen entsteben, ein V.organg, den ich auch in der That ofter beobachten konnte. Da der Process an dem einzelnen Kern vermuthlich schnell abläuft, in seiner Gesammtheit aber, wie ich glaube, sich über das ganze Leben des Thiers erstreckt, zudem nach ungefälrer Schätzung etwa die Hälfte der zweikernigen Zellen schon während des embryonalen Lebens in ebenso viel Wochen wie später in Jahren gebildet wird, so ist es klar, dass die Untersuchung postembryonaler Stadien nur geringe Aussicht auf Erfolg hat. Ich habe mich daher auch wesentlich an embryonale Stadien gehalten und an Serien des Bruststranges drei Wochen alter Kaninchenembryonen zahlreiche Bilder gesehen, die ich nur als Ausdruck direkter Kerntheilung deuten kann. Statt vieler gebe ich in Fig. $5 \mathrm{~b}, \mathrm{c}, \mathrm{d}$ drei besonders dentliche Abbildungen. Allerdings ist bei diesen Untersuchungen vor der voreiligen Diagnose einer Kerntheilung zu warnen. Denn anch abgeseben davon, dass nicht jede Unregelmässigkeit in der Form des Kerns 
oder selbst Einschnuirung ein sicheres Zeichen fuir direkte Theilung ist, wird man häufig durch theilweise sich deckende Kerne im ersten Angenblick versucht, eine Kerntheilung anzunehmen, die thatsächlich nicht vorhanden ist. Aber selbst unter Ausschluss aller zweifelhaften Fälle habe ich doch so viele evidente Kerntheilungen gesehen, dass ich den Process als zu recht bestehend ansehen muss.

Fragen wir uns nun zum Schluss nach der Bedeutung des Vorganges, so miissen wir mit Schwalbe die Anuahme, dass es sich um eine Hemmungsbildung im Sinne einer unvollkommenen Zelltheilung handelt, zuriickweisen. Die Thatsache, dass mit vorschreitendem Alter des Thieres die Zahl der zweikernigen Zellen auf Kosten der einkernigen zunimmt, macht es höchst unwahrscheinlich, dass die zweikernigen Zellen Uebergangsstadien darstellen, in denen der Theilungsprocess plötzlich unterbrochen wurde und za einem von nun an persistirenden Zustand führt. Es wäre sehr merkwärdig, wenn derselbe hemmende Eintluss bei embryonalen, in voller Entwicklung begriffenen Zellen in gleicher Weise sich geltend macht wie hei fertig ausgebildeten Zellen alter Thiere. Zudem sind Theilungen ausgewachsener Ganglienzellen unter normalen Verhältnissen bisher mit Sicherlicit nicht beobachtet worden. Es spricht vielmehr alles dafuir, dass wir die zweikernigen Zellen als das Ziel einer Entwicklung anzusehen haben, zu dem die einkernigen von vorne herein intendiren. Warum dieses Ziel das eine Mal schon im embryonalen Leben, das andere Mal erst in späteren Alter des Thieres erreicht wird, kann nur vermuthungsweise beantwortet werden.

Ganz unmöglich ist es, die Bildung der zwei Kerne als Ausdruck eines degenerativen Processes anzusehen, wie es für andere mehrkernige Zellen geschehen ist. Es erscheint ungereimt, einen Process furr degenerativ zu halten, der zum grossen Theil schon vor der Geburt zu einem stationären Zustand geführt hat, und dessen Intensität mit zunehmendem Lebensalter abnimmt. Auch steht mit der Annahme eines degenerativen Vorganges die Thatsache in Widerspruch, dass gerade die zweikernigen Zellen im Durchschnitt grössere Dimensionen annehmen als die einkernigen. Aus einer sehr grossen Anzahl von Messungen ergiebt sich, dass der Durchmesser der zweikernigen Zellen erwachsener Kaninchen c. $50 \mu$, der der einkernigen bei Kaninchen 
sowoll wie bei den Nagern, deren Zellen einkernig bleiben, nur c. $30 \mu$ beträgt.

Ich glaube daher, dass die Bildung der beiden Kerne in cinem innigen Zusammenhange mit den Wachsthumsverhältnissen der Zelle steht, der Art, dass die anfülngliche, uiberwiegende Ausbildung des Kernes zu einer Theilung desselben fübrt, welche ihrerseits die Veranlassung zu einem stärkeren Wachsthum der Zelle abgiebt. Ich vindicire also dem Process keine funktionelle, sondern lediglich eine biologische Bedentung fïr die Zelle.

\section{Erklärung der Abbildungen anf 'Tafel XXIII.}

Fig. 1. Einkernige bipolare Zelle mit mehreren Kapselkernen aus dem Grenzstrang eines vier Monate alten Kaninchens. Hartu. Obj. 7, Oc. 3.

Fig. 2. Einkernige multipolare Zelle aus dem Gangl. cerv. suprom. cines jungen Kaninchens. Hartn. Obj. 7, Oc. 3.

Fig. 3. Einkernige multipolare Zelle aus einem Lumbalgangl. eines zwei Jahre alten Kaninchens. Hartn. Obj. 7, Oc. 3.

Fig. 4. Dreikernige Zelle aus dem Gangl. coel. eines ausgewachsenen Kaninchens. Hartn. Obj. 7, Oc. 3.

Fig. 5. a Zweikernige Zelle aus dem Gangl. cerv. supr. eines dreiwöchentlichen Kaninchenembryos. Hartn. Obj. 7, Oc. 3. $b, c, d$ Kerntheilungen aus einem Dorsalgangl. eines dreiwöchentlichen Kaninchenembryos. Leitz Oel-Imm. 1/1, Oc. 3.

Fig. b̈. Zelle aus dem Gangl. cervic. supr. einer alten Maus. Hartn. Obj. 7 , Oc. 3 .

Fig. 7. Zwei Zellen aus dem Gangl. cervic. supr. eines viermonatlichen Hasen. Hartn. Obj. 7, Oc. 3.

Fig. 8. Zelle aus dem Gangl. cerv. supr. eines viermonatlichen Hasen. Hartn. Obj. 7, Oc. 3. 